Lessons Learned on X-ray Optics Fabrication: Work completed as part of the "Advancing the Technology R\&D of Tabletop Mesoscale Nondestructive Characterization" LDRD

M. J. Pivovaroff, W. W. Nederbragt, H. E. Martz

December 6, 2004 
This document was prepared as an account of work sponsored by an agency of the United States Government. Neither the United States Government nor the University of California nor any of their employees, makes any warranty, express or implied, or assumes any legal liability or responsibility for the accuracy, completeness, or usefulness of any information, apparatus, product, or process disclosed, or represents that its use would not infringe privately owned rights. Reference herein to any specific commercial product, process, or service by trade name, trademark, manufacturer, or otherwise, does not necessarily constitute or imply its endorsement, recommendation, or favoring by the United States Government or the University of California. The views and opinions of authors expressed herein do not necessarily state or reflect those of the United States Government or the University of California, and shall not be used for advertising or product endorsement purposes.

This work was performed under the auspices of the U.S. Department of Energy by University of California, Lawrence Livermore National Laboratory under Contract W-7405-Eng-48. 


\title{
Lessons Learned on X-ray Optics Fabrication: Work completed as part of the "Advancing the Technology R\&D of Tabletop Mesoscale Nondestructive Characterization" LDRD ${ }^{1}$
}

\author{
M. J. Pivovaroff, W.W. Nederbragt, H. E. Martz
}

\section{INTRODUCTION}

A Wolter X-ray optic was the central component of the microscope envisioned to fulfill the imaging requirements of the Characterization SI. After encountering many difficulties and delays, an optic was finally produced that, unfortunately, only partially met its specifications. With the SI halted, and efforts underway to reformulate a LDRD program to support fabrication of X-ray optics, it is useful to examine the previous effort and compile a list of lessons learned during the research.

\section{OVERVIEW}

It is extremely important to recognize that the goal of the project, begun as an LDRD ER and then folded into a larger LDRD SI, was never to develop the fabrication chain for manufacturing highresolution optics. Rather, it was to build an X-ray microscope and develop software to characterize mesoscale objects including NIF targets.[Martz, et al. 2003] And the optic represented only one component of the microscope. (Other important subsystems were the X-ray source, high-precision alignment stages, vibration and temperature control systems, an alignment methodology, and the detector). This situation required a very specific bottom-up approach to building the optic: perform a design study, establish the optical parameters (e.g., focal length and multilayer recipe), and then fabricate the optic ${ }^{2}$ using a pre-determined process adopted because of its success in the past. There was no R\&D planned because we were informed that making such a Wolter mirror would simply entail following an already demonstrated path for the construction of high-accuracy replicated optics.

\section{SUMMARY OF LDRD EFFORT}

The Characterization SI incorporated an on-going ER project tasked with building an X-ray microscope based on a Wolter X-ray optic. Initially, the only performance goal was achieving one micrometer spatial resolution over as large a FOV as possible with as much throughput as possible. The only external constraint was that the optic was to use multilayer coatings. These optical parameters, in turn, put very stringent requirements on the optic: the mirrors had to have an angular resolution of 0.4 arcsec (comparable to the performance of the Chandra X-ray telescope, which cost \$1 Billion) and the mirror surface had to have a roughness better than $5 \AA$.

As the mandrel manufacturing began, problems arose in a few different areas. Initially, vendors could not deposit the proper quality of nickel onto the mandrel to allow LLNL to accurately diamond turn the part. Once that problem was resolve, the next major difficulty was that another vendor could not polish the mandrel to the required level ( $5 \AA$ ) of surface roughness. This leads to a crucial realization: regardless of past performance, always verify through the use of coupons and independent

\footnotetext{
${ }^{1}$ This work was performed under the auspices of the U.S. Department of Energy by the University of California, Lawrence Livermore National Laboratory under Contract No. W-7405-Eng-48.

${ }^{2}$ Recall that the optic is made in three main steps: first a mandrel is produced, next the multilayers coatings are deposited on the mandrel, and finally a thick nickel coating is deposited on top of the multilayers. The thick nickel structure with the multilayers on the interior is then removed from the mandrel, leaving an unsupported X-ray optic.
} 
metrology that a vendor can meet specification and require both raw and analyzed data be included as a deliverable.

As delays were encountered, team members began contemplating the types of errors that might be introduced by vendors and the affect they would have on the performance. This work led to the development of a complete process flow for making an X-ray optic. The basic steps are shown in Figure 1. This top-down approach is completely different than that adopted for construction of X-ray optic under the LDRD — and would have required substantially more resources than were allocated. Specifically, the error budget and parameterized model was not defined or quantified at the beginning of the LDRD ER. To do so would have required many iterative cycles of fabricating and measuring parts, determining errors and their impact on performance, and then refining processes, a task requiring substantial investment.

Another significant by product of this work was forming relationships with other institutes with X-ray optics expertise. We had constructive conversations with the team at Zeiss that built the mirrors for

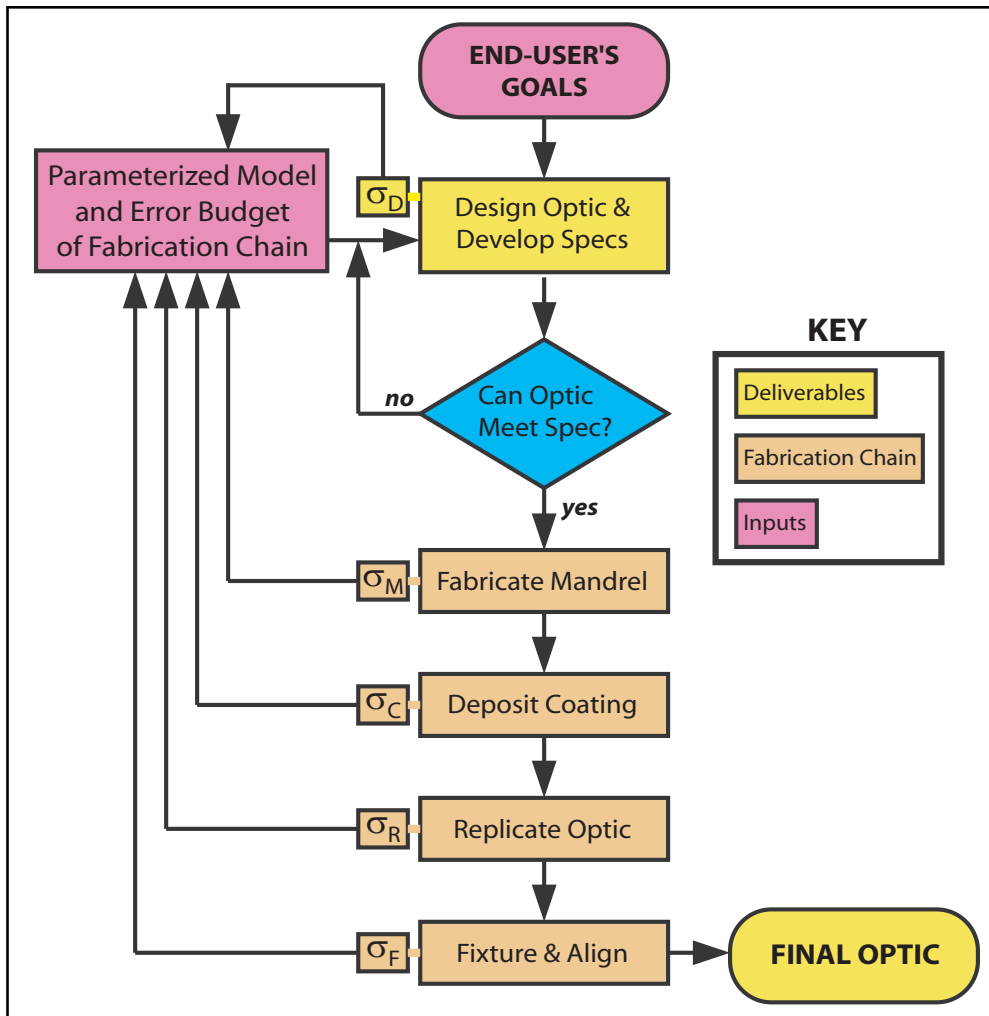

Figure 1: Replicated X-ray optic fabrication process flowchart several X-ray astronomy telescopes and Wayne McKinney of LBL who oversaw the fabrication of reflective mirrors for the Advanced Light Source. Our most productive interactions, however, were with a team of experts at NASA Marshall Space Flight Center (MSFC). MSFC have spent several years manufacturing replicated optics and have provided excellent insight into the factors that influence the success of the replication step and ways to predictably control the figure by adjusting the plating bath. We have begun collaborations with them to understand the errors from the nickel replication steps.

\section{THE TOP-DOWN APPROACH}

To understand the value of the top-down approach, consider the specific issue of the failure of the vendor to polish the mandrel to the required smoothness. The original specification was a smoothness of $\sigma \leq 5 \AA$, and the inferred value (based on X-ray measurements made at DSRI ${ }^{3}$ ) was $\sigma \geq 10.5 \AA$. How does this apparently modest change impact the performance of the optic? The multilayer roughness conforms to that of the mandrel, and the multilayer roughness intimately controls the reflectivity of the coatings. Thus, the throughput dropped from a nominal $16 \%$ to under $0.018 \%$, a $\sim 1000 \times$ decrease!

\footnotetext{
${ }^{3}$ Danish Space Research Institute
} 
If the project had the resources to follow the top-down approach, witness samples would have been sent to the vendor, the quality of the polish inserted into the error budget and process flow, and the use of multilayers would have been reconsidered. In this case, a different optical design could have been adopted that relied on a simple coating of gold or iridium that would be insensitive to a surface roughness of $\sigma \geq 10.5 \AA$ without a loss in throughput. Instead, resource constraints dictated adopting a particular bottom-up approach that had the multilayer coatings as a fixed requirement.

\section{SI ACHIEVEMENTS}

Although the X-ray optic produced had poor resolution ( $\sim 320 \operatorname{arcsec}$ instead of 0.4 arcsec) and low throughput, a major accomplishment was the deposition of an ambitious multilayer coating that is graded both laterally in depth, the first known application of such a structure in X-ray optics. This was verified by coating two super-polished silicon strips (roughness $\sigma=2 \AA$ ) with the multilayers that were deposited on the mandrel. These samples were then measured at the DSRI X-ray calibration facility at the same time the optic was characterized. Figure 2 plots the measured reflectivity against the best-fit model and shows the incredibly agreement between the two.

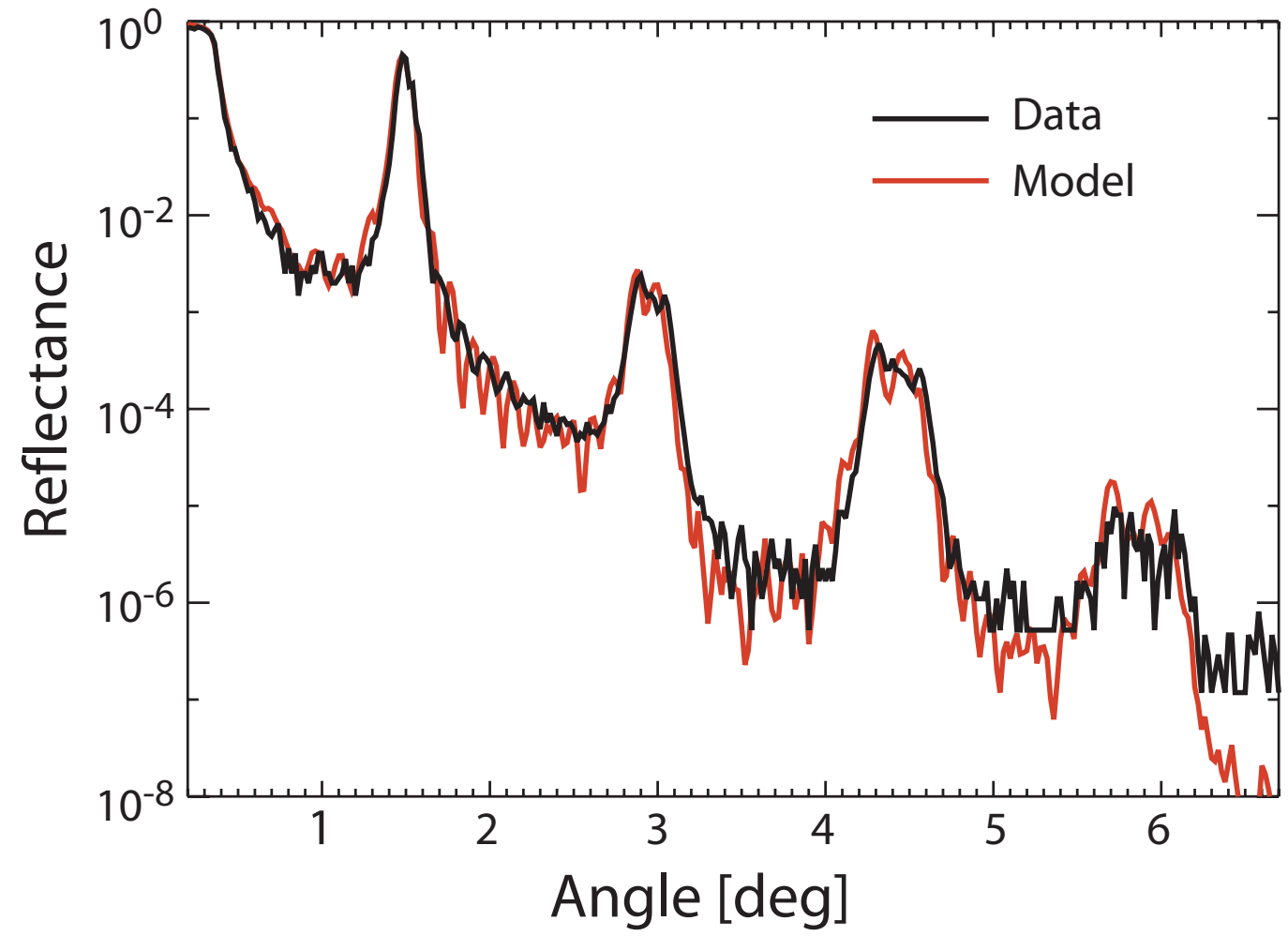

Figure 2: Measured reflectivity of the multilayer coating as a function of incident angle.

The measured roughness is $\sigma=2.5 \pm 0.2 \AA$, implying that if the optic had been polished to proper specification, the deposition process would have only slightly increased the multilayer roughness. For the test coatings, the peak reflectivity exceeds the $40 \%$ requirement ${ }^{4}$, leading to a predicted throughput of $20 \%$. Detailed analysis of these data are in preparation and will be published in Fall 2004.

\footnotetext{
${ }^{4}$ The throughput is determined by the square of the reflectivity, as the optic focuses X-rays with two mirror surfaces. Thus, a nominal reflectivity of $40 \%$ leads to a throughput of $[40 \% \times 40 \%]=16 \%$.
} 


\section{CONCLUSIONS}

Although the X-ray optic produced during the SI would not have met the ambitious goals of obtaining one micrometer spatial resolution, the team developed several important deliverables and realizations:

- It is crucial to continually work with previously established vendors to ensure their capabilities have not degraded to a level that they cannot meet specifications.

- LLNL now has a strong collaborative relationship with NASA MSFC. MSFC will provide valuable experience with the developing and improving the replication steps, essential for the achieving high spatial resolution.

- Engineering and PAT researchers have developed an initial framework for top-down manufacturing approach that incorporates a detailed process flow and error budget. Any future LDRD work must continue refinement of this, and work to characterize the state-of-art capabilities to accurately predict the performance of the next optics produced.

- Depth- and laterally-graded multilayers will perform as designed, enabling high-throughput systems.

These four important lessons are crucial for the success of future research and developments that LLNL may undertake in the near future in the area of high-precision X-ray optics manufacturing.

\section{REFERENCES}

Martz, Jr., H.E., J.H. Satcher, Jr., M.B. Aufderheide, T.W. Barbee, Jr., H.N. Chapman, J.A. Koch, W.W. Nederbragt, D.J. Schneberk, R.M. Sharpe, G.F. Stone, M.J. Pivovaroff, (2003) "Advancing the Technology R\&D of Tabletop Mesoscle Nondestructive Characterization," Lawrence Livermore National Laboratory, Livermore, Calif., UCRL-PROP-149198-REV-2. 